\title{
Femtosecond and UV inscribed grating characterization in photonic crystal fibres: optimization for sensing applications
}

\author{
Charalambos Koutsides *a,b, Eleni Yiangou ${ }^{a}$, Christos Themistos ${ }^{\mathrm{c}}$, Michael Komodromos ${ }^{\mathrm{c}}$, \\ Paul Christodoulides ${ }^{\text {a }}$, Kyriacos Kalli ${ }^{* * a}$ \\ ${ }^{a}$ Nanophotonics Research Laboratory, Department of Electrical Engineering / Computer \\ Engineering and Informatics, Cyprus University of Technology, Limassol, 3036, Cyprus \\ ${ }^{\mathrm{b}}$ Photonics Research Group, Aston University, B4 7ET, Birmingham, UK \\ ${ }^{\mathrm{c}}$ Frederick University, Filokyprou 7, Nicosia 1036, Cyprus
}

\begin{abstract}
Photonic crystal fibres (PCF) and more commonly microstructure fibres, remain interesting and novel fibre types and when suitably designed can prove to be "ideal" for sensing applications, as the different geometrical arrangement of the air holes alters their optical wave-guiding properties, whilst also providing tailored dispersion characteristics. This impacts the performance of grating structures, which offer wavelength encoded sensing information. We undertake a study on different air hole geometries and proceed with characterization of fibre Bragg and long period gratings, FBG and LPG, respectively that have been inscribed (using either a femtosecond or ultraviolet laser system) within different designs of microstructured fibre that are of interest for sensing applications.
\end{abstract}

\section{INTRODUCTION}

In the last decade the design and development of microstructured and/or photonic crystal optical fibres have flourished as a result of their tailored wavelength response, leading to potential in many fields and particularly in sensing applications [1-3]. In PCF, the core is shielded by an air hole geometry that defines fibre's characteristics; any changes in the geometry strongly influence the guided electromagnetic field. PCF can be designed to be single mode for a wide range of wavelengths compared with conventional fibre, whereas special geometries can provide high birefringence to these types of waveguides. Furthermore, as a result of the absence of rigid boundaries at the core-cladding interface, light travels in the same material minimizing material dispersion effects; this plays a strong influence on the wave-guidance of conventional optical fibres. These interesting characteristics turn PCF to critical components in new generation optical sensing applications like gas sensing, bio-sensing etc. [4]. The use of fibre Bragg and long period gratings in PCF further shift sensing applications to a new level, as the measurement information is wavelength encoded, offering an absolute measurement quantity. It is widely recognized that the inscription and characterization of UV and femtosecond fibre Bragg gratings (FBG) and long period gratings (LPG) in such fibres is of great importance. We have already demonstrated the femtosecond inscription of fibre Bragg gratings in custom made highly birefringence PCF specially designed for sensing applications [5].

Our work in this paper is divided into three steps. Initially, a modal analysis is presented in order to evaluate the bound modes for a number of fibre structures that are deemed suitable for grating inscription, or are of general interest as PCF, using either UV or femtosecond lasers. The different geometries are investigated and guided modes presented.

Email:*c.koutsides@cut.ac.cy **kyriacos.kalli@cut.ac.cy

Microstructured and Specialty Optical Fibres, edited by Kyriacos Kalli, Alexis Mendez,

Proc. of SPIE Vol. 8426, 84261O - (C) 2012 SPIE · CCC code: 0277-786X/12/\$18 - doi: 10.1117/12.925662

Proc. of SPIE Vol. 8426 842610-1 
Here we will utilize the full vectorial finite element method (FEM), which is applied in order to ascertain the effective refractive indices. In a second step, we present a theoretical study on how air holes geometries affect the optical properties of the fibres. Our main goal here is to track any birefringence changes for two different air hole patterns. As a result of the structure's complexity, non uniform meshing has been used for optimum area coverage of the cross section. In a third step, FBGs and LPGs have been introduced in PCF and their response is presented. The grating extent, with regard to cross-sectional area, is controlled to simulate the greatly confined refractive index changes that are created with a femtosecond laser, where non-linear absorption leads to a small focal volume. This contrasts with ultraviolet (UV) laser inscribed gratings that occupy the complete core cross section. Hence, we investigate the FBG reflectivity proportional to the size of the planes (grating width) that have been laser-inscribed within the core. We also consider the phase matching condition for LPG in PCF, where we systematically investigate the dispersion curves, thereby revealing how the grating period is modified in proportion to the resonant wavelength. Finally, we fill holes with liquid in order to investigate LPG response as are suitable for refractive index sensor based devices.

\section{MODAL ANALYSIS FOR DIFFERENT PCFS}

As we have already mentioned, the air hole geometries strongly influence supported modes for a PCF. Additionally, the number of the air hole rings surrounding the core of the fibre increases the refractive index contrast and as a result the mode confinement within the core increases. That impacts the fibre's guiding properties and influence grating's response. Because of the high complexity and the low symmetry of the structures, non uniform mesh applied in order the accuracy of our analysis to be enhanced.
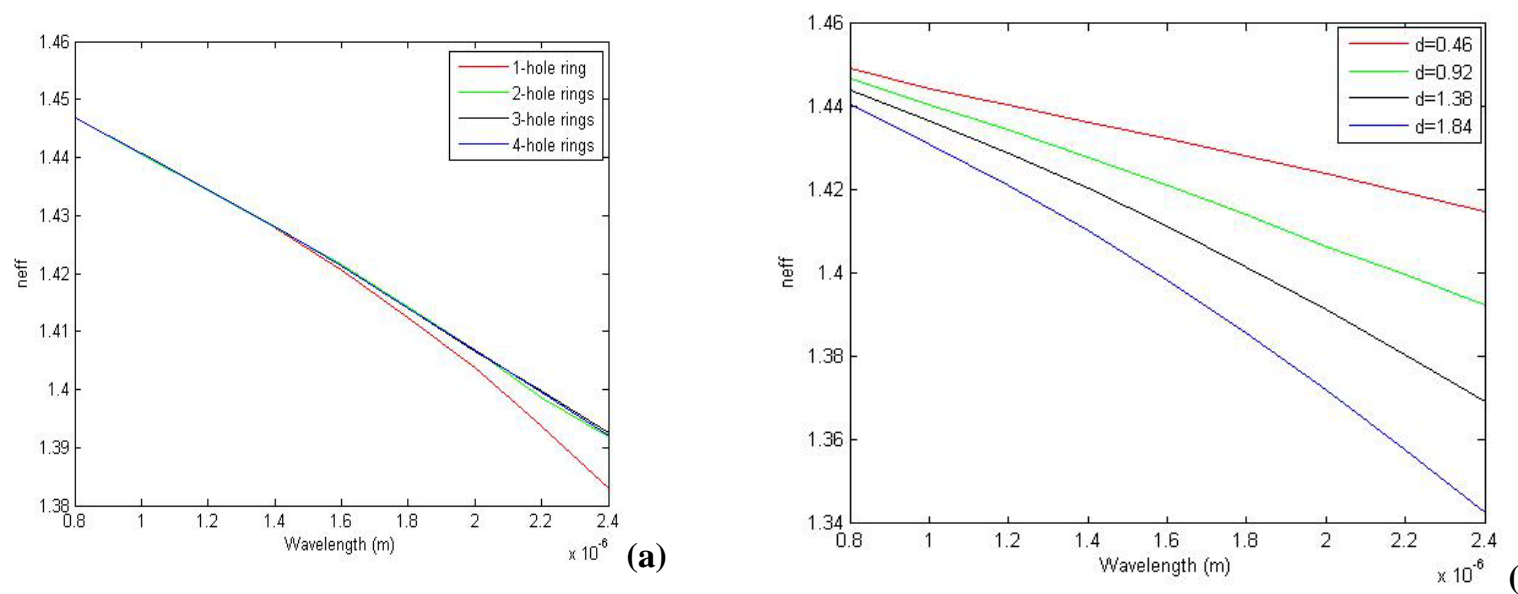

(b)

Figure 1. (a) Dispersion curves (effective refractive index with wavelength) for different number of air hole rings, (b) dispersion curves for a four rings air hole structure. The variable at this plot is the diameter of the air holes.

Figures above present the dispersion curves for different PCF structures. Figure 1(a) present the effective refractive indices for structures with one, two, three and four air hole rings. As the number of the air rings increases the mode confinement within the core increases, and as a result dispersion curves coincide. Furthermore, the difference between the curves increases for wavelengths with higher values; as the wavelength increases more light penetrates throughout the air holes and escapes to the outer cladding. For a single air hole ring structure, the light spreads for higher wavelengths and mode confinement reduces more quickly compared to the multi-ring structures.

In figure 1(b) the variable is the diameter of the air holes. The structure consists by four rings and the distances between the air holes remain constant. Filling factor control is achieved by controlling the diameter of the holes. As we can see, as the diameter of the air hole rings increases, more light is trapped in the cladding, contributing to the 
effective refractive index of the structure. We observe the results are wavelength sensitive and the curves diverge faster for greater wavelength values.

\section{BIREFRINGENCE IN PCF}

One of the main advantages of the PCF is the great potential for flexibility in their design. Sophisticated designs with lower degrees of symmetry, along orthogonal axis directions lead to highly birefringent PCF, but also having low confinement losses. The specially designed hole patterns around the core, with lower symmetry proportional to the orthogonal axis introduce birefringence that can be greater than two orders of magnitude compared with conventional optical fibres. Confinement losses remain low as a result of the high refractive index contrast between air holes and fibre's host material.

Here we will investigate and compare the birefringence between two different types of PCF; a standard endlessly single mode fibre (ESM-1550) with four air hole rings arranged in a hexagonal symmetry and a special designed PCF that consists of ten smaller air holes sandwiched between two rows of bigger holes (see figure 2)
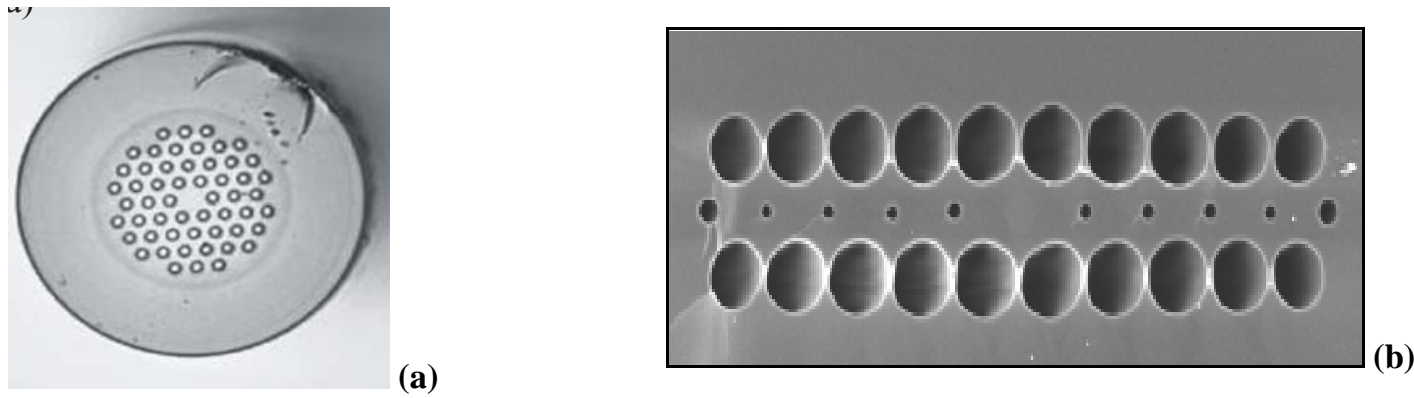

Figure 2. (a) Four air hole rings arranged in a hexagonal symmetry (ESM-1550), (b) ten smaller air holes sandwiched between two rows of larger air holes. Lower symmetry provides increased polarimetric sensitivity.

The beam propagation method (BPM) has been implemented in order for the birefringence to be calculated for each structure. In order to calculate the birefringence, the effective refractive indices have been defined for launch fields that are orthogonally polarized. As the BPM is sensitive to the step size along propagation direction (z), the $\mathrm{z}$ steps are set to be smaller by a factor of two than the filling factor for each structure. As PCF are inherently leaky structures, the correlation method has been implemented in our numerical analysis. The correlation method uses two full BPM propagations in order to solve for the modes of a structure. In the first propagation, the mode spectrum is found. From this spectrum, the eigenvalues, or neff values for each mode are extracted. Finally, in the second simulation the mode profiles, or eigenfunctions, are found. Figure 3 shows the analysis for the ESM-1550; for circular holes inducing birefringence of the order of magnitude is $10^{-6}$ (black line). As the holes becomes more elliptical the birefringence of the fibre increases; when the width of the hole is twice the height (ratio $=0.5$ blue line) the birefringence is ten times greater and the gradient of the graph increases. Here we have to mention that only the shape of the first two rings around the core has an important impact on our results.

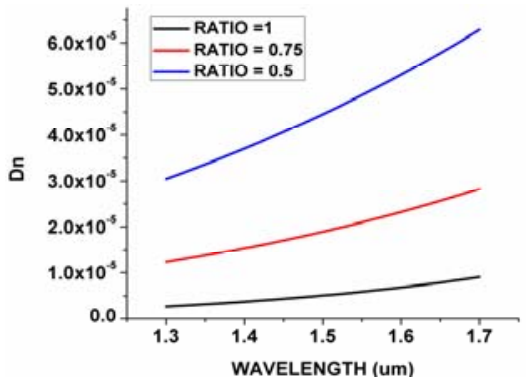

Figure 3. Effective refractive index difference for orthogonal direction proportional to free space wavelength. Black line is for circular air holes, Red line is for air holes with a ratio of 0.75 and Blue line refers to elliptical air holes with a ratio of 0.5. 
In order to develop a more suitable structure for sensing applications we selectively alter the shape of the surrounding air holes. As we can see from figure 4, two rings that are attached to the core are elliptical with a ratio of 0.75 . Despite the fact that the birefringence of the fibre remains at a very low level, a turning point appears at close to $1.55 \mu \mathrm{m}$ and this is promising for sensing applications, particularly for any gratings that coincide with this wavelength.
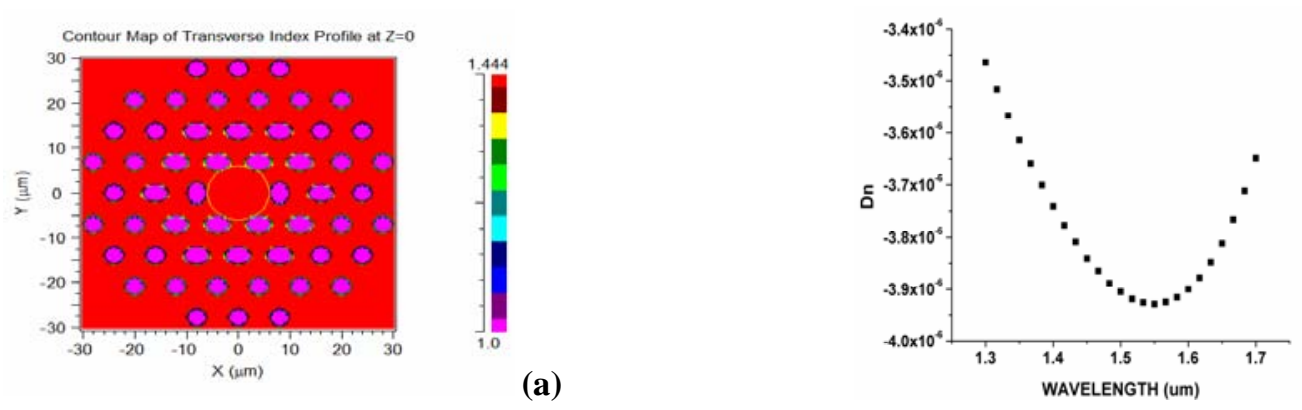

(b)

Figure 4. a) Two surrounding rings consisting of elliptical holes that have an aspect ratio of 0.75 . Other holes are circular, (b) effective refractive index difference showing a turning point around $1550 \mathrm{~nm}$.

For special applications that require stain and temperature measurements to be decoupled, a highly birefringent PCF has been considered. As we observe from figure 5, the birefringence of the custom made fibre is three orders of magnitude greater compared with ESM-1550. This is consistent with the design philosophy of the fibre. Furthermore, based on our observations for ESM-1550 we also alter the shape of the air holes in order to track any changes or possible turning points in birefringence values for that structure. In this case, we observe a lack of turning points, and also that the birefringence of the fibre is reduced as the structural symmetry is further disturbed. Finally, we observed that as the elliptical ratio of the air holes increases, confinement losses increases as a result of the reduction of the refractive index contrast along the vertical direction; more light leaks out from the core.

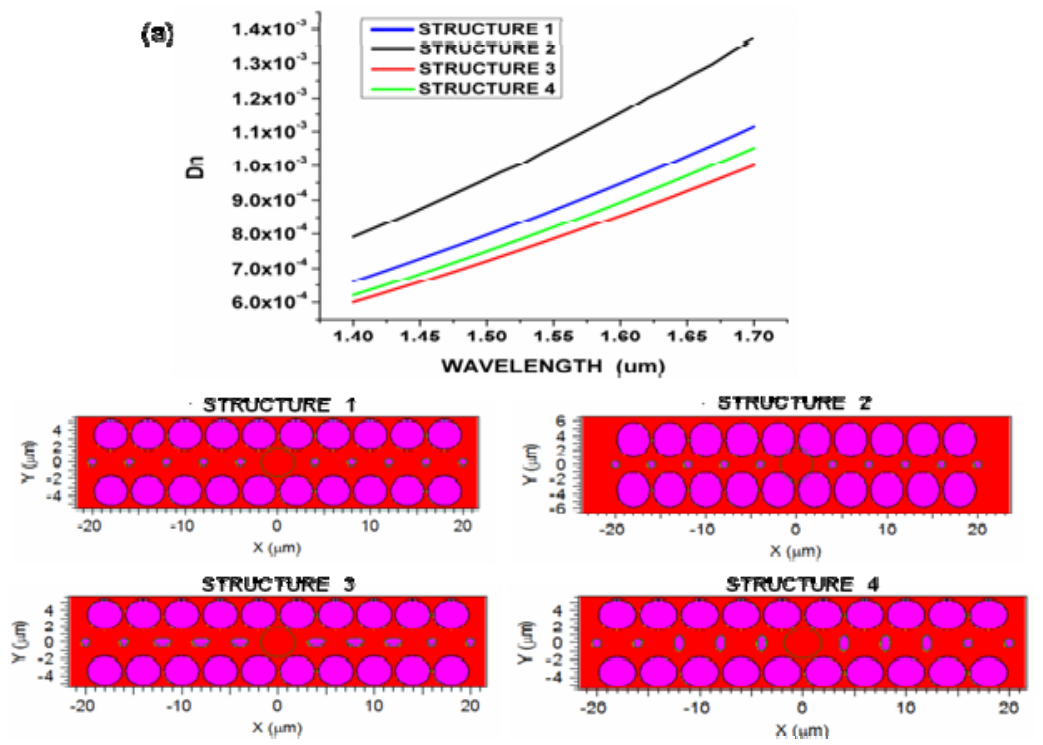

Figure 5. (a) Effective refractive index difference between the two orthogonal directions proportional to wavelength,

(STRUCTURE 1) the ratio between the vertical axis of the big holes is equal to 1, (STRUCTURE 2) the ratio between the vertical axis of the big holes is equal to 0.75 , (STRUCTURE 3 ) the ratio between the vertical axis of the small holes is equal to 2 , (STRUCTURE 4) the ratio between the vertical axis of the small holes is equal to 2 .

According to the above results, Structure 2 proves to be the highest birefringent structure (Black line); all the air holes of that structure are circular. As the air holes with the greater diameter become elliptic birefringence of the fibre drops and confinement losses increases (Blue line). Finally in order to further disturb fibre's geometry, in structures 3 and 4 the sandwiched air holes also become elliptical (Red and Green line respectively). Despite the fact 
that now the symmetry of the structures is minimum, birefringence further drops stating that the maximum birefringence with lower confinement losses correspond to structure 2

\section{FBG AND LPG IN PCF}

Laser inscribed gratings can be produced either by using UV or femtosecond lasers. The physical mechanisms that underlie each process are different as the femtosecond laser inscription is a non linear process. In UV inscription, the diffraction pattern introduces refractive index changes that occupy the whole diameter of the core. On the other hand, as femtosecond laser inscription is a non-linear process implemented via multi-photon absorption, the refractive index changes are confined within the focal volume of the beam; grating spots can be smaller than core's diameter. For this analysis we assume that gratings are uniform and located along the fibre axis so that maximum symmetry is achieved, as this has a great impact on the calculation times.

Figure 6 shows the effective refractive indices and the reflectivity for a UV and a femtosecond inscribed FBG. The effective refractive indices are slightly different for the two cases, whereas the reflectivity for the femtosecond inscribed grating is approximately ten times smaller. The smaller cross-section of the femtosecond inscribed grating results in an interaction with a portion of the mode. Any power distributed in the mode wings does not interact with the structure. On the other hand, as UV inscribed gratings occupies the whole diameter of the core the interaction of the structure with the grating is maximal and the reflectivity significantly increases

(a)

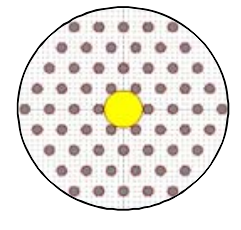

(d)

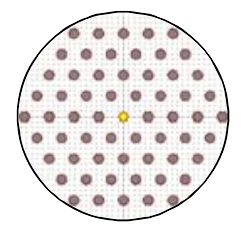

(b)

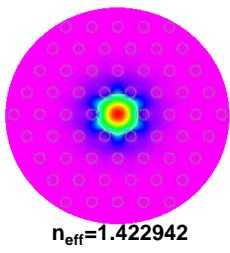

(e)

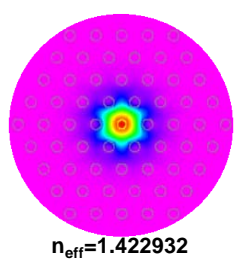

(c)

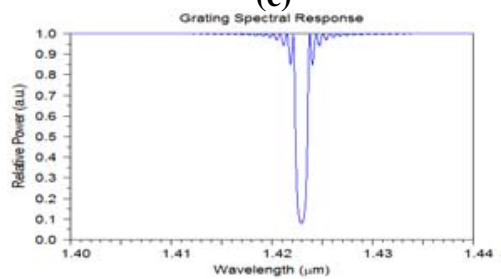

(f)

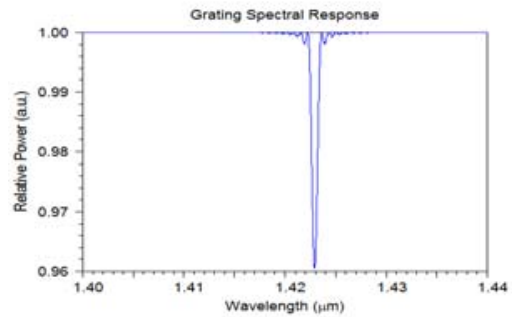

Figure 6. (a) and (d) are the index profile for UV (3.6 $\mu \mathrm{m}$ cross-section width) and femtosecond ( $0.8 \mu \mathrm{m}$ cross-section width) inscribed Bragg gratings, respectively, (b) and (e) are the calculated modes with their effective refractive indices, (c) and (f) are the respective transmission spectra, assuming the same index change for the gratings

The ability to tailor the grating reflectivity is essential for a number of special types of gratings, such as sampled gratings, hence we further investigate their response in proportion to the cross section of the grating (we term this as Grating Width). Femtosecond-laser inscribed FBG with different cross section and UV-inscribed gratings have been introduced in the different PCF. As the air hole geometries alter the optical properties of the fibre, gratings have been introduced into the PCF and their response is presented in figure 7(a). 

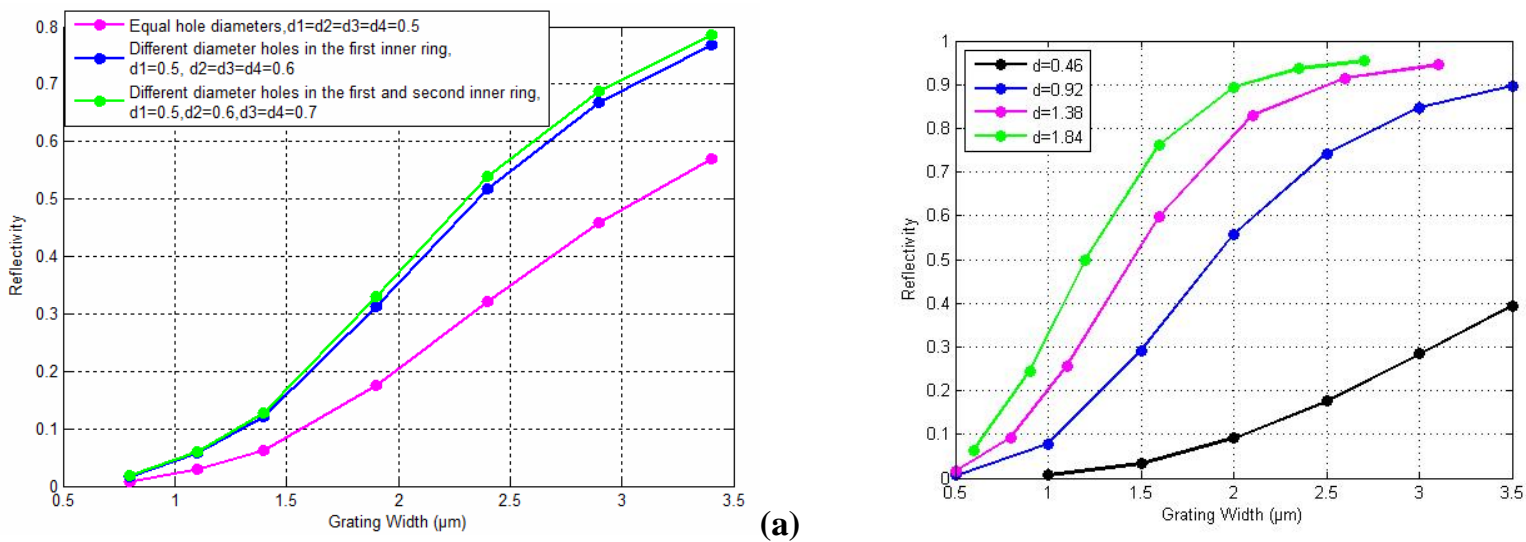

(a)

(b)

Figure 7. (a) Gratings reflectivity inscribed in PCF with different hole diameters, (b) grating reflectivity inscribed within PCF having different filling factor. The distance between air-holes $(\Lambda)$ remains constant.

As the diameter of air holes increases, the refractive index contrast is enhanced and the mode has greater confinement within the core. As a result, the reflectivity of the grating, for a given grating width, increases. Furthermore, the gradient of the graph is steeper for larger air holes, as the power distribution is narrower and the grating acquires a greater "intensity". Finally, we increase the air-hole diameters for two rings around the core in order to study possible changes (green line). As we see from the above figure, the contribution of the second air hole ring is minor and for a $2-\mu \mathrm{m}$ grating width across the core, the change remains below $5 \%$. Another design parameter that influences the grating's response is the filling factor of the fibre; the filling factor determines the effective refractive index and spatial power distribution of the modes. Figure 7(b) shows that as the filling factor increases the reflectivity of the grating significantly increases. For example, reflectivity of a $2-\mu \mathrm{m}$ grating width inscribed across the core in a PCF, having a filling factor of 0.8 (green line), is a approximately nine times greater than the reflectivity of the same grating inscribed within PCF with a filling factor 0.2 (black line). Finally, as the grating cross-sectional width increases, the reflectivity obeys a power law.

For further investigation of grating's response LPG have been introduced in the PCF. The long period grating is a periodic refractive index modulation of the material of the core with a period of several hundred micrometers. The long period grating couples the light travelling within core with resonant co-propagating cladding modes creating attenuation bands in the transmitted spectrum. The core mode is coupled to the resonant cladding mode that satisfies the phase matching condition shown in the equation below.

$$
\lambda=\left(n_{c o}^{e f f}-n_{c l}^{e f f}\right) \Lambda
$$

where $n_{c o}^{e f f}$ is the effective mode index of the core, $n_{c l}^{e f f}$ is effective mode index of the cladding, $\lambda$ is the resonance wavelength and $\Lambda$ is the grating period. As the resonance wavelength is sensitive to several parameters such as temperature, strain, bending and more other parameters, LPG have been widely used as sensitive optical sensors. Furthermore, as we can see from the equation above, effective refractive index of the cladding strongly influences the resonance wavelength of the grating. The PCF fibres can have a variable $n_{c l}^{e f f}$ as the air holes can be filled with liquids or gasses; this offers great flexibility and serves to increase their potential in sensing applications. Finally, special geometries of PCF can overcome cross sensitivity phenomena that inherently limit the accuracy of a measurement. Figure 8 shows the index profile of the fibre under investigation. The effective refractive indices of the core and cladding have been calculated for a range of wavelengths using the finite element method (FEM). The grid step is set to be $0.5 \mu \mathrm{m}$, which is sixteen times smaller than the distance between the hole centres, whereas the filling factor is $\mathrm{d} / \Lambda \approx 0.46$. The material dispersion has been calculated using the Sellmeier equation and has been included in our calculations. Finally, the fibre's diameter is set to be $125 \mu \mathrm{m}$. As the same grating can couple light to several different modes at different wavelengths, the graph below is a phase matching representation demonstrating the relationship between grating period and resonance wavelength. 

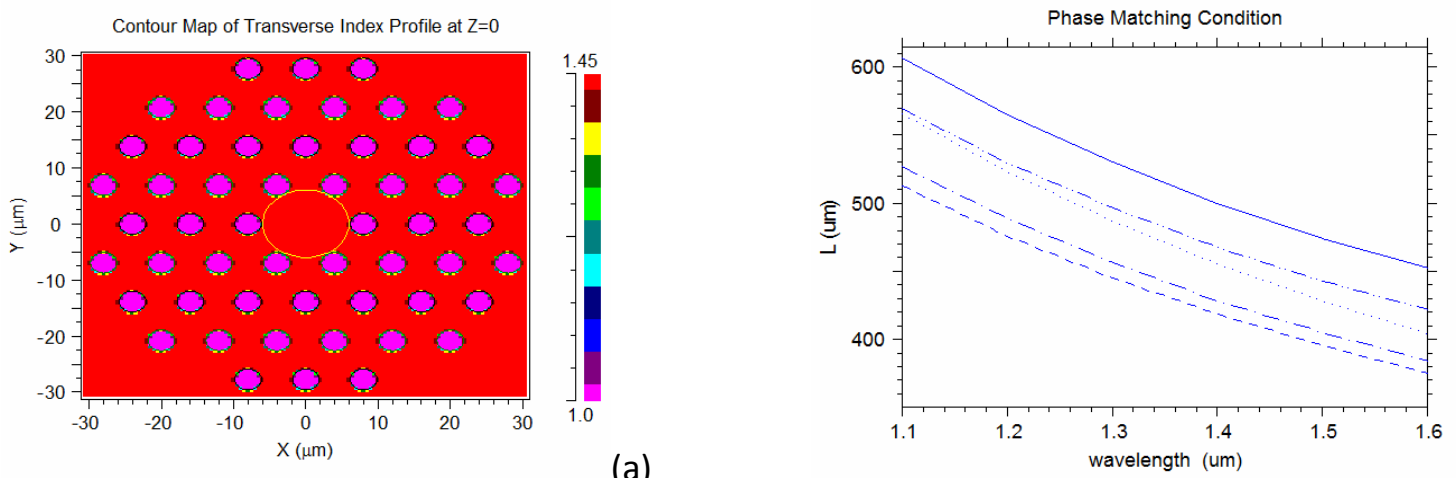

(b)

Figure 8: LPGs period required for core - cladding coupling proportional to wavelength. Mode order and grating are inverse proportional.

(a)

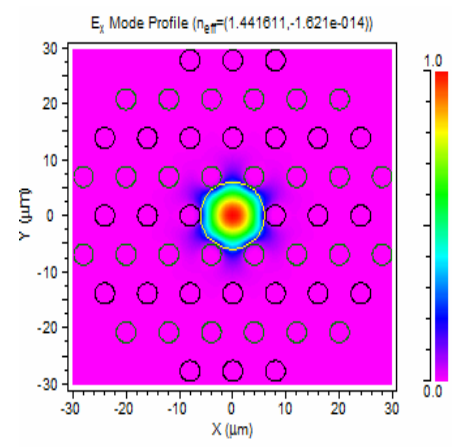

(d)

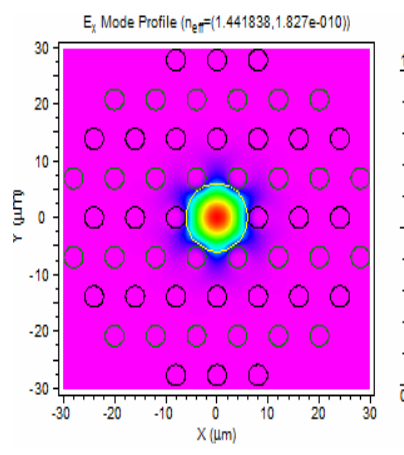

(b)

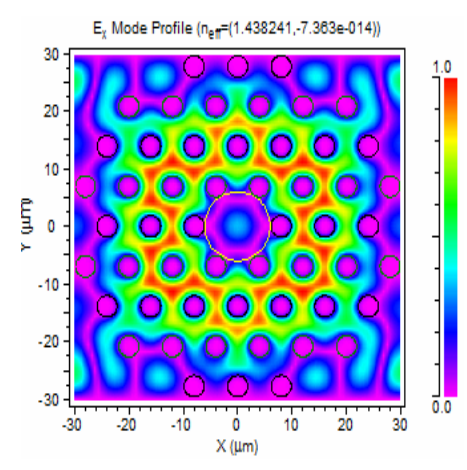

(e)

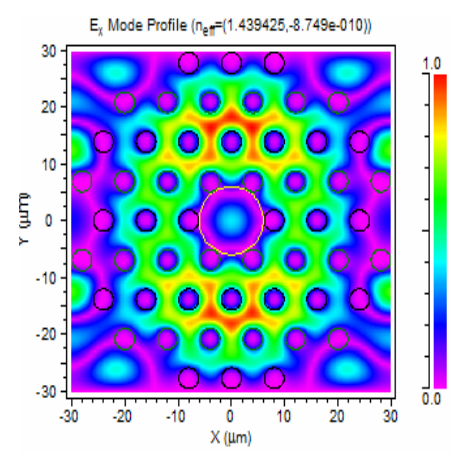

(c)

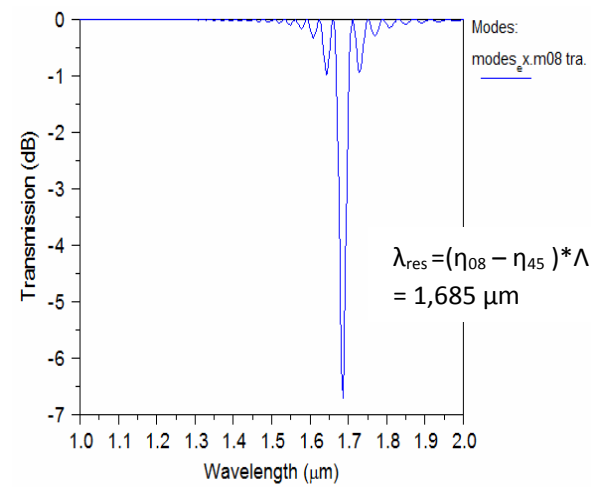

(f)

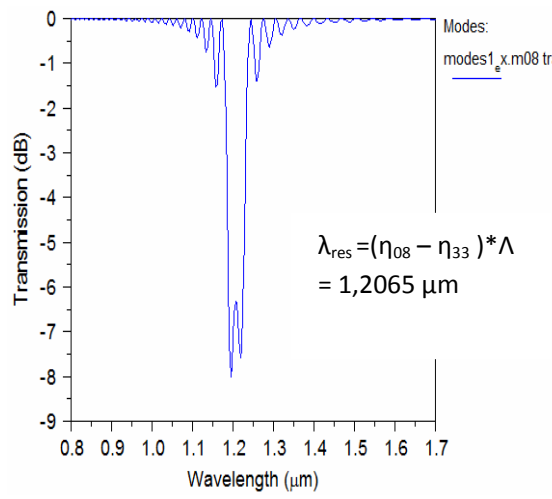

Figure 9. (a) and (d) Core mode when holes are filled with air $(\mathrm{n}=1)$ and liquid $(\mathrm{n}=1.33)$ respectively, (b) and (e) dominant cladding mode when holes are filled with air $(n=1)$ and liquid $(n=1.33)$ respectively, $(c)$ and $(\mathrm{f})$ transmission spectra when holes are filled with air $(\mathrm{n}=1)$ and liquid $(\mathrm{n}=1.33)$ respectively.

As we can see the same grating meets the phase matching condition for more than one wavelength; higher mode orders create resonances for shorter wavelengths, and the dispersion is responsible for the negative sign of the slope. As the guided wavelength increases, more light penetrates the air holes giving rise to the material dispersion. Considering that the effective refractive index of air is smaller than silica, the effective refractive indices of the guided modes decreases. As a result, the core-cladding effective refractive index difference increases faster than the wavelength. 


$$
\Lambda=\frac{\lambda}{\delta \eta_{e f f}}
$$

Thus, the grating period required for the phase matching condition gets smaller explaining in turn the negative sign of the slope that appears in the dispersion diagram (Equation (2)). The phase matching condition was used to construct the grating characteristic that shows the required beat length as a function of wavelength. Resonant modes of the LPG with period $L$ could be found in intersections of the grating characteristic with the line $\Lambda=L$. Since the solution is not necessarily unique, an additional criterion is required. Here, the cladding mode that maximizes the coupling coefficient is chosen as resonant [6]. As the coupling coefficient is determined by a scalar product of the electric fields of the interacting modes, modes with parallel field vectors are most likely to be resonant. Finally, we are compare the LPG response in cases where holes contain air and when holes are filled with a liquid (refractive index, $\mathrm{n}=1.33$ ). The period of the grating is $500 \mu \mathrm{m}$ and the filling factor is 0.48 . Initially, the core mode and the dominant cladding mode have been calculated for the two cases (see figure 9). Effective refractive indices are different for the two filling materials as we expected; this impacts the transmission spectra as the phase matching condition depends on these values. The resonance wavelengths are validated as they agree with the values that are calculated using the phase matching condition (Equation (1)). Furthermore, as the liquid fills the holes, the dominant cladding mode becomes a lower order mode, whereas birefringence is introduced to the fibre. Finally, the reflectivity of the grating drops marginally as less power is confined within the core; due to the lower refractive index contrast.

\section{CONCLUSION}

We have applied the FEM and coupled mode theory to investigate the optical properties of different geometries of PCF and to describe the transmission spectra of femtosecond and UV inscribed gratings. Our results show that changes in the fibre's geometry strongly influence the grating response. Additionally, we show that as the grating cross-sectional width increases, for femtosecond inscribed gratings, the reflectivity obeys a power law. Reflectivity control is crucial for many applications, and different grating types, such as sampled gratings. This further promotes the idea that femtosecond-laser inscription as a flexible technique for grating development with tailored characteristics. Finally, we present that LPGs can be used for refractive index sensing applications.

\section{REFERENCES}

1. J. C. Knight, T. A. Birks, P. St. J. Russell, and D. M. Atkin, "All-silica single-mode optical fibre with photonic crystal cladding," Opt. Lett. 21, 1547-1549 (1996).

2. Chengkun Chen, Albane Laronche, Géraud Bouwmans, Laurent Bigot, Yves Quiquempois, and Jacques Albert, "Sensitivity of photonic crystal fibre modes to temperature, strain and external refractive index", Optics Express, Vol. 16, Issue 13, pp. 9645-9653 (2008)

3. Ren Guobin, Wang Zhi, Lou Shuqin, and Jian Shuisheng, "Mode classification and degeneracy in photonic crystal fibres", Optics Express Vol. 11, No. 11, (2003)

4. Shu-Guang Li, Si-Ying Liu, Zhao-Yuan Song, Yin Han, Tong-Lei Cheng, Gui-Yao Zhou, and Lan-Tian Hou, "Study of the sensitivity of gas sensing by use of index-guiding photonic crystal fibres", Applied Optics, Vol. 46, Issue 22, pp. 5183-5188 (2007)

5. T. Geernaert, K. Kalli, C. Koutsides, M. Komodromos, T. Nasilowski, W. Urbanczyk, J. Wojcik, F. Berghmans, and $\mathrm{H}$. Thienpont, "Point-by-point fibre Bragg grating inscription in free-standing step-index and photonic crystal fibres using near-IR femtosecond laser," Opt. Lett. 35(10), 1647-1649 (2010).

6. A. Othonos and K. Kalli, "Fibre Bragg Gratings". Norwood, MA: Artech House, 1999 